Review

\title{
Xenobiotic Metabolism: The Effect of Acute Kidney Injury on Non-Renal Drug Clearance and Hepatic Drug Metabolism
}

John Dixon ${ }^{1,2}$, Katie Lane ${ }^{1,2}$, Iain MacPhee ${ }^{2,3}$ and Barbara Philips ${ }^{1,2, *}$

1 General Intensive Care Unit, St. George's Hospital, London SW17 0QT, UK;

E-Mails: johndixon3@nhs.net (J.D.); klane@sgul.ac.uk (K.L.)

2 Division of Clinical Sciences, St. George's, University of London, London SW17 0RE, UK

3 Renal Medicine, St. George's Hospital, London SW17 0QT, UK; E-Mail: imacphee@sgul.ac.uk

* Author to whom correspondence should be addressed; E-Mail: bphilips@sgul.ac.uk; Tel.: +44-20-8725-5071; Fax: +44-20-8725-3296.

Received: 9 December 2013; in revised form: 12 December 2013 / Accepted: 27 December 2013 / Published: 13 February 2014

\begin{abstract}
Acute kidney injury (AKI) is a common complication of critical illness, and evidence is emerging that suggests AKI disrupts the function of other organs. It is a recognized phenomenon that patients with chronic kidney disease (CKD) have reduced hepatic metabolism of drugs, via the cytochrome P450 (CYP) enzyme group, and drug dosing guidelines in AKI are often extrapolated from data obtained from patients with CKD. This approach, however, is flawed because several confounding factors exist in AKI. The data from animal studies investigating the effects of AKI on CYP activity are conflicting, although the results of the majority do suggest that AKI impairs hepatic CYP activity. More recently, human study data have also demonstrated decreased CYP activity associated with AKI, in particular the CYP3A subtypes. Furthermore, preliminary data suggest that patients expressing the functional allele variant $C Y P 3 A 5^{*} 1$ may be protected from the deleterious effects of AKI when compared with patients homozygous for the variant $C Y P 3 A 5^{*} 3$, which codes for a non-functional protein. In conclusion, there is a need to individualize drug prescribing, particularly for the more sick and vulnerable patients, but this needs to be explored in greater depth.
\end{abstract}

Keywords: acute kidney injury; cytochrome P450; drug metabolism; pharmacogenetics; pharmacokinetics; CYP3A 


\section{Introduction}

The liver is the organ responsible for the majority of drug metabolism through the actions of the cytochrome P450 (CYP) enzyme group (Phase 1 reactions) and the enzymes of conjugation (Phase 2 reactions) [1]. Although these enzymes are predominantly active within the liver, many may also be found in various other organs including, kidney, gastrointestinal tract and lung [2]. Their function within these tissues may be of great importance to the function of that organ but with the exception of enzymes within the gastrointestinal wall, it is thought that overall drug metabolism is predominantly determined by the liver enzymes because of their abundance. Cytochrome P450 activity within the gut may have a marked effect on the bioavailability of orally administered medicines, less so on parenterally administered drugs.

Drug elimination however, occurs mainly via the kidneys [1]. This may be the parent drug itself or active or non-active metabolites and excretion predominantly occurs through filtration of hydrophilic compounds. However, there may also be significant secretion and re-absorption of compounds within the renal tubules, the importance of which varies between drugs and may change markedly in critical illness [3]. Other organs involved in the elimination of drugs include; the liver (through biliary excretion), the gastro-intestinal tract, lung and skin.

Drug dosing in AKI (acute kidney injury) is usually based upon empiric principles or is extrapolated from data obtained from patients with chronic kidney disease (CKD). This rationale, however, is flawed because AKI and CKD are different clinical entities with different etiologies and, for example, AKI occurring in the context of multi-organ dysfunction [4] has a different inflammatory milieu to CKD. Causes of AKI are outlined in Table 1. Furthermore, the time course for disease progression and adaptability differs which may result in varying pharmacokinetic and pharmacodynamic responses to the same drug in time: the rapidly changing nature of AKI means that therapeutic drug concentrations may not be achieved, or, alternatively, drug toxicity may ensue. Nevertheless, some mechanistic processes may be shared between CKD and AKI. AKI is inherently difficult to study, so, interesting results from investigations in patients with CKD may be useful in informing targets for research in AKI. The impact of CKD on non-renal clearance, particularly upon the function of CYP enzymes is now well recognized [5-7] and recommendations for drug dosing and interactions have been adjusted accordingly by the Food and Drug Administration (FDA) [8] The activity of the CYP enzymes may be similarly impaired in critically ill patients [3,9] and evidence suggests that acute kidney injury (AKI) may be implicated [10,11]. This review will focus on the evidence of the impact of AKI on non-renal drug clearance, with particular reference to CYP activity and expression.

\section{The Kidney as a Metabolic Organ}

Although the kidney is largely thought of as the organ responsible for elimination of waste products, toxins and drugs from the body, it is in fact more complex and has many other functions. These include; water and electrolyte homeostasis, maintenance of plasma osmolarity, acid-base balance, and the production and secretion of hormones, e.g., renin, erythropoietin, 1,25-dihydroxyvitamin D3 [12,13]. Some functions may be more important in pathological states [4], such as catabolism of peptide hormones and gluconeogenesis in fasting conditions. The kidneys receive $25 \%$ of the cardiac 
output under normal conditions and have high oxygen needs, largely caused by the energy required for the reabsorption of sodium ions in the proximal tubules [14].

As renal function declines, each of the kidney's functions is affected, including clearance of drugs and their metabolites, although different functions may decline at different rates.

Table 1. Causes of acute kidney injury (AKI).

\begin{tabular}{ccc}
\hline Category of AKI & Mechanism & Causes \\
\hline $\begin{array}{c}\text { Pre-renal failure } \\
\text { Renal hypoperfusion }\end{array}$ & Hypovolaemia/Hypotension & $\begin{array}{c}\text { Haemorrhage, dehydration (diarrhoea and } \\
\text { vemiting, heat), Osmotic diuresis (hyperglycaemia, } \\
\text { iatrogenic), excessive diuretic use }\end{array}$ \\
& Poor cardiac function & $\begin{array}{c}\text { Sepsis, anaphylaxis, reduced plasma oncotic } \\
\text { pressure in nephrotic syndrome, pancreatitis } \\
\text { Cardiogenic shock, severe sepsis, }\end{array}$ \\
Renal vascular changes & Afferent arteriolar vasoconstriction \\
Intrinsic-renal failure & (NSAIDs, ACE inhibitors, vasoconstrictors)
\end{tabular}

\section{Importance of AKI (Acute Kidney Injury)}

AKI is a clinical syndrome, defined in recent Kidney Disease Improving Global Outcomes (KDIGO) guidelines as "an abrupt decrease in kidney function that includes, but is not limited to, acute renal failure" [15]. AKI is defined according to three stages of severity: Stage 1 is defined by a rise in serum creatinine ( $\mathrm{SCr}$ ) of $>1.5$ times the baseline over the preceding seven days, a rise $>26.4 \mu \mathrm{mol} / \mathrm{L}$ over the previous 2 days, or $<0.5 \mathrm{~mL} / \mathrm{Kg} / \mathrm{h}$ urine output for $>6 \mathrm{~h}$; Stage 2 is defined by $\mathrm{SCr}>2.0$ the baseline, or urine output $<0.5 \mathrm{~mL} / \mathrm{Kg} / \mathrm{h}$ for $12 \mathrm{~h}$; and Stage 3 is defined by SCr greater than three times the baseline, initiation of renal replacement therapy, increase of $\mathrm{SCr}$ to $>354 \mu \mathrm{mol} / \mathrm{L}$ or urine output $<0.3 \mathrm{~mL} / \mathrm{Kg} / \mathrm{h}$ for $>24 \mathrm{~h}$ or anuria for $>12 \mathrm{~h}$. An important feature of KDIGO criteria is that it defines AKI by relatively small increases in SCr. Retrospective observational cohort studies of patients with AKI reveal that small increases in $\mathrm{SCr}(>26.4 \mu \mathrm{mol} / \mathrm{L})$ are associated with increased mortality when compared with patients without a change in renal function or patients with CKD [16]. The fact that relatively small changes in renal function affect mortality adds strength to the proposal that increased mortality is not merely due to retention of uraemic toxins, and that an underlying pathophysiological process may be affecting mortality in AKI. 
There are several limitations to creatinine-based definitions of AKI; the most important are reduced sensitivity in patients with CKD [17] and reduced formation of SCr in critically ill patients [18].

Within critical care units, hypo-perfusion is the commonest cause of AKI [19], resulting in a mixture of pre renal failure and intrinsic AKI due to acute tubular necrosis (ATN). Once AKI develops, patients may experience some clinical effects generic to all causes of AKI, regardless of its aetiology, in addition to any effects specific to the illness causing AKI in that individual. It has not yet been established, however, whether different etiologies of AKI result in different clinical effects predominating.

AKI is a common complication of critical illness, and AKI requiring renal replacement therapy alone accounts for approximately $9 \%$ of all bed-days in general adult critical care units [20] and considerably more if all severities of AKI are included. Mortality from AKI requiring renal replacement therapy is between $43.3 \%$ and $74.5 \%$ [20,21] and has remained unchanged over the last 40 years, despite advances in renal replacement therapy. The reasons for the poor outcome from AKI are unclear. It is possible that the poor outcome is due to the decreased renal excretion of drugs and toxins. Decreased excretion may be directly attributable to a reduced glomerular filtration rate (GFR), reduced tubular secretion or impaired renal metabolism of drugs. There is, however, emerging evidence that AKI also affects the clearance of drugs and toxins by other organs (i.e., non-renal clearance; $C l_{\mathrm{NR}}$ ). These may contribute to high mortality associated with AKI. The evidence for this will be evaluated later and placed into clinical context.

\section{Pharmacokinetics in AKI}

There is a general paucity of pharmacokinetic (PK) studies concerning drugs in AKI and it is suggested that this may be due to a lack of incentive by pharmaceutical companies to fund studies in AKI because this is not yet a requirement of the FDA [10]. This complicates our understanding of such changes and in critically ill patients this is further complicated by multi-organ effects and cross talk between organs. Pro and anti-inflammatory changes, kidney, liver and endothelial dysfunction, drug interactions, therapeutic interventions, perfusion abnormalities, intestinal atrophy or gut dysmotility (impairing absorption of enterally administered drugs) are amongst the many confounding factors affecting the PK of any drugs administered in critically ill patients [9]. The volume of distribution of a drug may be affected by changes to cardiac output and peripheral perfusion [22]; and depends upon the degree of protein binding, tissue permeability and lipid solubility [23]. Other influential factors during AKI include changes to blood $\mathrm{pH}$, the impact of AKI on $\mathrm{pKa}$ of the drug and the effects of fluid shifts between body compartments [24]. Each of these aspects may be influenced by renal dysfunction.

Currently, most drug-dosing regimens in patients with AKI are extrapolated from patients with CKD and of the evidence from studies of PK in AKI most are obtained from animal studies or are ex vivo investigations using cell cultures or microsomal systems. Interestingly it is suggested that in vitro studies using homogenates of renal cells may vastly underestimate the impact of AKI on drug metabolic pathways. Drug metabolizing enzymes are sited regionally within the kidney, for example; CYP enzymes are situated within the renal cortex, prostaglandin synthase within the medulla, $\mathrm{N}$-acetyl transferase within both cortex and medulla [25]. Homogenizing a whole kidney means that enzyme activity may be grossly underestimated. 
AKI has many etiologies, each with differing effects, e.g., Gentamicin accumulation within the renal cortex results in toxicity [26], causing acute tubular necrosis, whereas Cyclosporine toxicity causes altered renal haemodynamics and vasoconstriction [27]. It is possible that different etiologies of AKI have differing effects on hepatic enzyme activity.

\section{Organ Crosstalk}

Non-renal clearance $\left(C l_{\mathrm{NR}}\right)$ in AKI differs from that observed in patients without renal impairment. Even drugs normally associated with predominantly renal clearance (e.g., vancomycin) appear to be impacted upon by change in $C l_{\mathrm{NR}}$ in AKI [10] although the mechanisms are unclear. A possible explanation for non-renal effects of AKI is "organ crosstalk", that is, in the case of the kidneys, the effect of AKI on the function of other organs. Organ crosstalk has been defined as "the effects of one malfunctioning organ upon the function of another" [28], and is usually associated with injurious effects. An often studied example is that of acute lung injury following AKI. AKI results in increased pulmonary vasculature permeability to albumin, erythrocyte sludging in lung capillaries, interstitial edema and an inflammatory cell infiltrate in affected lung tissue [29]. Interestingly, these changes have been observed in rat models following kidney-ischaemia reperfusion injury [30] but not following bilateral nephrectomies [31], perhaps supporting the argument that this phenomenon is not purely due to uremia alone. Inflammation may contribute to remote organ dysfunction in AKI and may be important in the development of AKI. Animal models of ischemic AKI have demonstrated an abundance of cell adhesion molecules, increased cytokine-chemokine expression, leukocyte trafficking, dysregulation of apoptosis and increased oxidative stress in distant organs [31].

\section{Potential Mechanisms of How AKI Affects Non-Renal Drug Clearance}

The major contributor to $C l_{\mathrm{NR}}$ is clearly hepatic clearance $\left(C l_{\mathrm{HEP}}\right)$ determined by the following equation [32]:

$$
C l_{\mathrm{HEP}}=\left[Q \times\left(C l_{\mathrm{INT}} \times f_{\mathrm{UB}}\right)\right] /\left[Q+\left(C l_{\mathrm{INT}} \times f_{\mathrm{UB}}\right)\right.
$$

$Q=$ liver blood flow, $C l_{\mathrm{INT}}=$ intrinsic hepatic clearance and $f_{\mathrm{UB}}=$ fraction of unbound drug

AKI may theoretically impact on each aspect of $C l_{\text {HEP. }}$. Changes to liver blood flow affect uptake and elimination of drugs with a high extraction rate [33]. How important this is in renal-hepatic cross talk is uncertain as the full impact of AKI on liver blood flow and the liver micro-circulation is unknown. What is known is patients with CKD [34] and sepsis [35] have preserved global hepatic blood flow, perhaps suggesting it is not the most important factor.

Changes to protein binding and, hence the unbound fraction of a drug, have been observed in critically ill patients, thus altering the amount of drug available for metabolism within the liver [36]. Decreased serum albumin is initially a consequence of redistribution into interstitial fluids and later contributed to by decreased synthesis. Albumin binds mainly neutral and acidic drugs [37]. An important example is phenytoin which is highly bound to albumin, but its free fraction is increased in trauma patients as the serum albumin decreases [3]. The direct impact of AKI on serum albumin concentrations is uncertain but given that AKI is a pro-inflammatory state it is likely that it has some effect [37]. 
The usual rate-limiting step of intrinsic hepatic clearance is CYP activity [1]. CYP3A is responsible for metabolism of over $50 \%$ of drugs and there are two predominant isoforms CYP3A4 and CYP3A5. Individuals who possess at least one allele for the wild type $C Y P 3 A 5^{*} 1$ gene (i.e., both homozygotes and heterozygotes) produce a functional protein. People homozygous for $C Y P 3 A 5^{*} 3$ produce a non-functioning CYP3A5 protein which is the predominant form in $80 \%$ of Caucasian populations. The wild type predominates in Sub-Saharan Africans [38], 60\% are homozygous for $C Y P 3 A 5 * 1$. Other CYP enzymes involved in drug metabolism in humans include CYP1A, CYP2C19, CYP2C9 and CYP2D6, together they account for $80 \%$ of all drug metabolism [39]. CYP3A activity is impaired in CKD [40] and in AKI [11,41,42]. The impact of renal impairment on other CYP enzymes remains uncertain and may vary.

The underlying mechanisms for the inhibition of CYP enzymes are obscure. Parathyroid hormone, urea, and cytokines are all proposed as potential mediators [43]. Serum fractionation experiments in rodents suggest a 10-15 kDa substance may be responsible [44], but its identity is yet to be established.

Although CYP activity is responsible for the majority of drug metabolism, other enzyme systems have a role.

Flavin-containing-mono-oxygenase (FMOs) oxidizes xenobiotics containing Nitrogen, Sulfur, or Phosphorus [45]. They catalyze some of the same reactions as CYP enzymes [46], although they often result in different metabolites with potentially different pharmacological actions. The physiological functions of FMOs are currently poorly understood. Genetic polymorphism has been observed in three of the five human expressed FMO genes, FMO1, FMO2 and FMO3 [45]. The consequences of the genetic polymorphisms on drug metabolism remain poorly understood. The impact of AKI on FMO gene expression and FMO activity remains unclear.

There have been no studies directly investigating the effect of AKI on mono-amine oxidase (MAO), however, administration of pargyline, an irreversible MAO inhibitor, to a rat model of renal ischemia-reperfusion resulted in decreased tubular apoptosis and necrosis and increased proximal tubular cell proliferation [47]. This study demonstrated a central role of MAO in mediating the production of reactive oxygen species, which contribute to ischemia-reperfusion injury.

We have been unable to find in vitro, animal, or human studies investigating the impact of AKI on other enzyme systems, such as alcohol dehydrogenase, epoxide hydrolase, prostaglandin synthase or conjugation (Phase II) pathways.

Hepatic drug clearance also depends on drug transport systems including; organic anion transporter proteins (OATPs) which control uptake of drugs into hepatocytes [10] and P-glycoprotein which facilitates the elimination of drugs and metabolites from hepatocytes into the bile or blood. This is discussed in greater detail in Section 8. The impact of AKI on these systems remains unclear.

\section{Evidence for Kidney-Liver Crosstalk}

Experiments performed in animal models of AKI suggest a significant effect of AKI upon the inflammatory response and subsequent hepatic function. An altered balance of anti-inflammatory cytokines (e.g., IL-4, IL-10) and pro-inflammatory cytokines (e.g., TNF- $\alpha$, IL-1, IL-6) and neutrophil infiltration has been observed in mice [36,48-52], rats [53,54] and dogs [55] following renal ischaemia-reperfusion injury and bilateral nephrectomy. IL-6 is a possible mediator of down-regulation 
of CYP activity [56]. In hepatic cell culture studies, several CYP isoforms have been down-regulated by IL-6 [57], but the clinical implications of this remain untested. AKI causes IL-6 to increase earlier and faster in sepsis [58], following cardiac surgery [59] and during acute lung injury [60]. IL-6 also activates the hypothalamo-pituitary axis, causing increased cortisol, which is an endogenous substrate of CYP enzymes and may lead to competitive inhibition [61]. This effect, however, is difficult to test because of the variability of cortisol in the critically ill, and because increased cortisol may induce CYP3A synthesis. Decreased activity of superoxide dismutase and catalase (both anti-oxidants) and increased malondialdehyde and transaminases concentrations have also been observed, implying that some of the observed changes in the liver may be due to oxidative stress [54].

The majority of animal studies investigating CYP activity demonstrate reduced hepatic drug metabolism, particularly when investigating CYP3A activity, however, some disparities exist. No change in Clarithromycin [62] or Telithromycin [63] metabolism via CYP3A was observed following Uranyl nitrate-induced AKI in rats, whereas decreased metabolism of Etoposide [64] and Losartan [65] metabolism via the CYP3A enzyme was demonstrated following Uranyl nitrate-induced AKI in rats. CYP3A activity was also reduced following Cisplatin-induced AKI in rats, as demonstrated by elevated Tacrolimus [66] and Quinine [67] concentrations. Gentamicin-induced AKI did not change CYP3A-mediated Cyclosporine metabolism in rats [68], whereas it was reduced following renal-ischaemia-reperfusion injury in rabbits [69]. AKI induced by bilateral ureteric ligation or by Uranyl nitrate did not result in altered CYP-2D6 mediated metabolism of Metoprolol in rats [70,71]; no change in CYP2D6-mediated metabolism of Propranolol was observed in rats when AKI was induced by Cisplatin [66], but elevated Propranolol concentrations were observed, implying reduced CYP2D6 activity, when AKI was induced by bilateral ureteric ligation in rats [72]. Increased metabolism of Theophylline, via the CYP2E1 enzyme, was observed following Uranyl nitrate-induced $\mathrm{AKI}$ in rats [73], and increased CYPD2C-mediated metabolism of Tolbutamide was observed following glycerol-induced AKI in rats [74].

AKI may induce modifications in the transcription and translation of CYP enzymes; however, changes in mRNA-CYP protein expression do not always result in altered enzyme activity. Decreased mRNA-directed expression of CYP2E1 was observed in AKI following renal-ischaemia-reperfusion-injury in rats [75], whereas it was increased following Uranyl nitrate-induced AKI [76].

It is difficult to directly apply data obtained from experiments in animals to humans. The apparent conflicting evidence may be due in part to the characteristics of the drugs studied and the model of AKI used. In addition, CYP isoforms are different between species and control of CYP expression may also differ. The changes in CYP activity that occur in one organ do not always occur in others (e.g., increased activity in intestine despite decreased activity of CYP3A4 in liver). While changes in hepatic drug metabolism was not always observed, it is possible that other pharmacokinetic changes may have occurred, for example, altered intestinal absorption or gut CYP3A activity, or altered protein-binding. Extrapolation of data from animal studies to humans requires extreme caution. 


\section{Transporters}

Few studies have been performed in AKI, and all are in animal models or cell cultures. Interspecies differences exist, in their tissue distribution and subtypes. These need to be taken into account when extrapolating data to humans.

Renal drug clearance involves transport across the basolateral and apical tubular membranes. Transporter activity is a major influence on drug clearance. The main mechanisms involve (1) passive diffusion through the lipid cell layer, or via aqueous channels; (2) Carrier mediated transport via either active (ATP-dependent) transport or facilitated transport.

Drugs passively diffuse across cell membranes along a concentration gradient without expenditure of energy. Important factors affecting lipid diffusion are: the concentration, the surface area of the drug (greater diffusibility occurs with drugs that have a larger surface area), and the lipid-solubility of the drug. The lipid-solubility depends on the lipid-aqueous partition co-efficient (i.e., how readily a drug can pass through a lipid membrane), the degree of ionization of the drug, which depends on the pKa of the drug and the $\mathrm{pH}$ of the surrounding cell medium. Aqueous diffusion occurs via aqueous pores along a concentration gradient. Drugs passing through aqueous pores are small (molecular mass $<30 \mathrm{kD}$ ) and water-soluble in solution. We have been unable to find data regarding the specific effects of AKI upon passive diffusion of drugs; however, it is possible that acidosis associated with AKI may impact on the lipid-aqueous partition co-efficient by altering $\mathrm{pH}$ of body fluids.

Carrier-mediated transport is important for drugs that are too large or too insoluble in lipid to diffuse through lipid membranes. Carriers are trans-membrane proteins and mainly located in renal tubules, the biliary tract, the blood-brain barrier and the gastrointestinal tract. Active transport occurs against a concentration gradient, is energy dependent with energy obtained from hydrolysis of ATP. Carriers are selective, may become saturated once a threshold has been reached, and may undergo competitive inhibition by another drug binding to the same receptor. One large family is the $\mathrm{ABC}$ (ATP binding cassette) and includes P-glycoprotein, or multidrug resistance type 1 (MDR1) transporter. P-gp is an ATP-dependent efflux pump expressed in the liver, kidneys and intestines [10]. It assists transportation of lipophilic compounds from inside cells to the bile, urine and intestinal lumen, assisting with clearance of the drug from the body. Increased P-gp expression was observed in the kidney of rats with AKI [77], but not their liver [78] or intestines [79]. Clearance of P-gp substrates was decreased, however, throughout the body, including via the liver, kidneys and intestines, implying global suppression of P-gp function during AKI. The effect of AKI on P-gp suppression may impact on clearance of drugs such as Digoxin, Methotrexate and Vincristine [80].

The organic anion transporters (OATs) and organic cation transporters (OCTs) are important in transferring drugs across cell membranes. OATs mainly occur in renal tubular basolateral membranes and enhance uptake of small organic anions from the peri-tubular plasma into renal tubular cells, by efflux across the apical membrane into the tubular lumen [10]. Decreased OAT-1 and OAT-3 mRNA protein expression was observed in rats with AKI [81]. The role of OATs in non-renal clearance has not yet been clarified; however, decreased activity during AKI may impact on clearance of drugs such as Methotrexate and NSAIDs [82]. We could find no literature regarding the effect of AKI upon organic cation transporter (OCT) activity. 


\section{Human Studies}

To date, three published studies have investigated the effect of AKI on hepatic drug metabolism in humans $[11,41,42]$. Heinemeyer investigated the effect of AKI upon hepatic clearance of Cetriaxone in post-operative patients with pneumonia and AKI [42]. Delayed biliary excretion was demonstrated, however, the free-drug fraction differed between patients with AKI and those without. The underlying mechanisms were not explored, so the authors were unable to exclude sepsis as the cause of liver dysfunction, rather than AKI. A second study by the same group investigated the clearance of monomethylaminoantipyrine (MMAAP) in critically ill adults with AKI [41]. Significantly reduced Clearance of MMAAP was observed in patients with AKI compared to those without AKI.

Furthermore, a significantly reduced rate of appearance of its metabolites $N$-formylaminoantipyrine and $\mathrm{N}$-acetylaminoipyrine was observed. It appears likely that the reduced hepatic metabolism was responsible for decreased rate of MMAAP clearance occurring in AKI, although the authors were unable to exclude other potential confounders, such as hypoxia, reduced cardiac output, reduced protein synthesis, or competitive antagonism by other drugs. However, in contrast to their previous study, less than $10 \%$ of subjects had septic shock, making sepsis unlikely as the sole cause of this phenomenon. More recently, our group has used an intravenous Midazolam probe to investigate CYP3A4 and CYP3A5 activity in critically ill patients with AKI [11]. A significant decrease in Midazolam elimination was observed in patients with AKI, and this effect appeared to be increased with prolonged durations of AKI. These findings were significant, despite the heterogenous population studied, perhaps implying a potent effect. Other potential confounders, such as acid-base balance and serum albumin concentration, were not significantly different between patients with AKI and those without. In addition, preliminary data suggests CYP function may be preserved in patients with AKI who expressed either homozygous or heterozygous functional allele variant $C Y P 3 A 5^{*} 1$ when compared with those who were homozygous for the splice variant $C Y P 3 A * 5$, which codes for a non-functional truncated protein. This could have important pharmacogenetic implications for patients with AKI but remains to be fully tested.

It is plausible that uraemic toxins may be responsible for the changes in CYP activity occurring during AKI, and it is also conceivable that removing potential toxins with renal replacement therapy or plasma exchange may reverse the non-renal clearance effects observed in AKI. To date, few studies have investigated this. In one study, patients with AKI had increased Telithromycin concentration and exposure (as measured by area under the curve), however, AUC approached that of healthy individuals within two hours of renal replacement therapy [83]. In another study, the 14C-Erythromycin breath test was used as a marker of CYP3A4 activity and 27\% increase in activity was observed $2 \mathrm{~h}$ after initiation of renal replacement therapy [84].

\section{Role of Intestinal Metabolism}

The majority of drug metabolism occurs in the liver, although metabolism within the gut wall is important for some orally administered drugs. CYP3A accounts for $80 \%$ of small intestine drug metabolism but only $1 \%$ of total body CYP3A activity [85]. Some drugs depend on gut wall enzymes to convert a pro-drug to its active form. Uptake from the gut lumen occurs either by diffusion or active 
OAT-mediated transport, before being then passed into the portal circulation en route back to the liver or extracted back into the gut lumen. It is not know to what extent AKI impacts on this process, however, critical illness in general may impair absorption by altering gut perfusion, it may impair gut motility and may alter gut flora. We were unable to find any data on the effects of AKI on intestinal CYP2J2, the other abundant intestinal CYP enzyme.

\section{Conclusions}

Current drug-dosing guidelines and regimens in patients with AKI have several limitations. The majority use empiric principles or use data extrapolated from patients with CKD. The pitfalls of this are becoming more obvious and we have got to the stage where greater understanding of the wider implications of AKI is required in order to optimize drug treatment of who are very often very vulnerable patients. It is likely that we should be making adjustments for alterations in hepatic drug clearance during AKI. The mechanisms for any effect on non-renal drug clearance remain unclear but involve the accumulation of toxins (e.g., IL-6).

Our understanding is limited by small studies and extrapolation of data from animal studies and in vitro investigations. Metabolizing enzymes and transporter isoforms differ between species and interpretation of data from immortal cells lines, particularly concerning the expression of certain proteins is fraught with problems. Nevertheless evidence is accumulating that, as with CKD, AKI does have a significant impact on the hepatic metabolism of drugs and could also affect drug clearance in other organs (e.g., intestines). Clinical studies investigating the effect of AKI may be confounded by the existence of multiple pathological processes in critically ill patients with AKI that may also impair CYP and transporter activity (e.g., sepsis, trauma, burns). It is also likely that, once the pharmacokinetic effects of AKI have been accounted for, inflammatory mediators occurring during critical illness may influence the pharmacodynamic response to drugs.

In order to bring this field forward and improve drug prescribing on individual level, future studies need to elucidate mechanisms at the enzyme and mRNA levels and their clinical effects in patients with and without AKI. Furthermore, distinctions need to be made between gut, liver and kidney metabolism of the various CYP enzymes and transporters. The influence of other co-existent diseases that contribute to AKI needs to be accounted for and excluded if possible. Identification of putative uraemic toxins, and whether their removal by renal replacement therapy improves non-renal clearance, also needs clarification.

\section{Conflicts of Interest}

The authors declare no conflict of interest.

\section{References}

1. O'Shaunessy, K. Principles of Clinical Pharmacology and Drug Therapy. In Oxford Textbook of Medicine, 5th ed.; Warrel, D., Cox, T., Firth, J., Eds.; Oxford University Press: Oxford, UK, 2010; Volume 1, pp. 1450-1476. 
2. Gardiner, S.J.; Begg, E.J. Pharmacogenetics, drug-metabolizing enzymes, and clinical practice. Pharmacol. Rev. 2006, 58, 521-590.

3. Smith, B.S.; Yogaratnam, D.; Levasseur-Franklin, K.E.; Forni, A.; Fong, J. Introduction to drug pharmacokinetics in the critically ill patient. Chest 2012, 141, 1327-1336.

4. Owen, O.E.; Felig, P.; Morgan, A.P.; Wahren, J.; Cahill, G.F., Jr. Liver and kidney metabolism during prolonged starvation. J. Clin. Investig. 1969, 48, 574-583.

5. Dowling, T.C.; Briglia, A.E.; Fink, J.C.; Hanes, D.S.; Light, P.D.; Stackiewicz, L.; Karyekar, C.S.; Eddington, N.D.; Weir, M.R.; Henrich, W.L. Characterization of hepatic cytochrome p4503A activity in patients with end-stage renal disease. Clin. Pharmacol. Ther. 2003, 73, 427-434.

6. Dreisbach, A.W.; Japa, S.; Gebrekal, A.B.; Mowry, S.E.; Lertora, J.J.; Kamath, B.L.; Rettie, A.E. Cytochrome P4502C9 activity in end-stage renal disease. Clin. Pharmacol. Ther. 2003, 73, 475-477.

7. Naud, J.; Michaud, J.; Beauchemin, S.; Hebert, M.J.; Roger, M.; Lefrancois, S.; Leblond, F.A.; Pichette, V. Effects of chronic renal failure on kidney drug transporters and cytochrome P450 in rats. Drug Metab. Dispos. 2011, 39, 1363-1369.

8. Dreisbach, A.W.; Lertora, J.J. The effect of chronic renal failure on drug metabolism and transport. Expert Opin. Drug Metab. Toxicol. 2008, 4, 1065-1074.

9. Boucher, B.A.; Wood, G.C.; Swanson, J.M. Pharmacokinetic changes in critical illness. Crit. Care Clin. 2006, 22, 255-271.

10. Vilay, A.M.; Churchwell, M.D.; Mueller, B.A. Clinical review: Drug metabolism and nonrenal clearance in acute kidney injury. Crit. Care 2008, doi:10.1186/cc7093.

11. Kirwan, C.J.; MacPhee, I.A.; Lee, T.; Holt, D.W.; Philips, B.J. Acute kidney injury reduces the hepatic metabolism of midazolam in critically ill patients. Intensiv. Care Med. 2012, 38, 76-84.

12. Bougle, A.; Duranteau, J. Pathophysiology of sepsis-induced acute kidney injury: The role of global renal blood flow and renal vascular resistance. Contrib. Nephrol. 2011, 174, 89-97.

13. Brown, D.; Wagner, C.A. Molecular mechanisms of acid-base sensing by the kidney. J. Am. Soc. Nephrol. 2012, 23, 774-780.

14. Ricksten, S.E.; Bragadottir, G.; Redfors, B. Renal oxygenation in clinical acute kidney injury. Crit. Care 2013, doi:10.1186/cc12530.

15. Group, K.A.W. KDIGO clinical practice guideline for acute kidney injury. Kidney Int. Suppl. 2012, 2, 1-138.

16. Tian, J.; Barrantes, F.; Amoateng-Adjepong, Y.; Manthous, C.A. Rapid reversal of acute kidney injury and hospital outcomes: A retrospective cohort study. Am. J. Kidney Dis. 2009, 53, 974-981.

17. Doi, K.; Yuen, P.S.; Eisner, C.; Hu, X.; Leelahavanichkul, A.; Schnermann, J.; Star, R.A. Reduced production of creatinine limits its use as marker of kidney injury in sepsis. J. Am. Soc. Nephrol. 2009, 20, 1217-1221.

18. Wilson, F.P.; Sheehan, J.M.; Mariani, L.H.; Berns, J.S. Creatinine generation is reduced in patients requiring continuous venovenous hemodialysis and independently predicts mortality. Nephrol. Dial. Transplant. 2012, 27, 4088-4094. 
19. Stewart, J.; Findlay, G.; Smith, N.; Kelly, K.; Mason, M. Adding Insult to Injury: A Review of the Care of Patients Who Died in Hospital with a Primary Diagnosis of Acute Kidney Injury (Acute Renal Failure). A report by the National Confidential Enquiry into Patient Outcome and Death (2009). Available online: http://www.ncepod.org.uk/2009report1/Downloads/AKI_report.pdf (accessed on 8 February 2014).

20. Kolhe, N.V.; Stevens, P.E.; Crowe, A.V.; Lipkin, G.W.; Harrison, D.A. Case mix, outcome and activity for patients with severe acute kidney injury during the first $24 \mathrm{~h}$ after admission to an adult, general critical care unit: Application of predictive models from a secondary analysis of the ICNARC Case Mix Programme Database. Crit. Care 2008, doi:10.1186/cc7003.

21. Abosaif, N.Y.; Tolba, Y.A.; Heap, M.; Russell, J.; El Nahas, A.M. The outcome of acute renal failure in the intensive care unit according to RIFLE: Model application, sensitivity, and predictability. Am. J. Kidney Dis. 2005, 46, 1038-1048.

22. Meier-Hellmann, A.; Reinhart, K.; Bredle, D.L.; Specht, M.; Spies, C.D.; Hannemann, L. Epinephrine impairs splanchnic perfusion in septic shock. Crit. Care Med. 1997, 25, 399-404.

23. Martyn, J.A.; Abernethy, D.R.; Greenblatt, D.J. Plasma protein binding of drugs after severe burn injury. Clin. Pharmacol. Ther. 1984, 35, 535-539.

24. Power, B.M.; Forbes, A.M.; van Heerden, P.V.; Ilett, K.F. Pharmacokinetics of drugs used in critically ill adults. Clin. Pharmacokinet. 1998, 34, 25-56.

25. Lash, L.H. Role of renal metabolism in risk to toxic chemicals. Environ. Health Perspect. 1994, 102, 75-79.

26. Whelton, A. Aminoglycoside renal cortical kinetics: A clue to mechanisms of nephrotoxicity. Prog. Clin. Biol. Res. 1979, 35, 33-41.

27. English, J.; Evan, A.; Houghton, D.C.; Bennett, W.M. Cyclosporine-induced acute renal dysfunction in the rat. Evidence of arteriolar vasoconstriction with preservation of tubular function. Transplantation 1987, 44, 135-141.

28. Lane, K.; Dixon, J.J.; MacPhee, I.A.; Philips, B.J. Renohepatic crosstalk: Does acute kidney injury cause liver dysfunction? Nephrol. Dial. Transplant. 2013, 28, 1634-1647.

29. Kramer, A.A.; Postler, G.; Salhab, K.F.; Mendez, C.; Carey, L.C.; Rabb, H. Renal ischemia/reperfusion leads to macrophage-mediated increase in pulmonary vascular permeability. Kidney Int. 1999, 55, 2362-2367.

30. Hassoun, H.T.; Grigoryev, D.N.; Lie, M.L.; Liu, M.; Cheadle, C.; Tuder, R.M.; Rabb, H. Ischemic acute kidney injury induces a distant organ functional and genomic response distinguishable from bilateral nephrectomy. Am. J. Physiol. Ren. Physiol. 2007, 293, F30-F40.

31. Serteser, M.; Koken, T.; Kahraman, A.; Yilmaz, K.; Akbulut, G.; Dilek, O.N. Changes in hepatic TNF-alpha levels, antioxidant status, and oxidation products after renal ischemia/reperfusion injury in mice. J. Surg. Res. 2002, 107, 234-240.

32. Wilkinson, G.R.; Shand, D.G. Commentary: A physiological approach to hepatic drug clearance. Clin. Pharmacol. Ther. 1975, 18, 377-390.

33. Nolin, T.D.; Naud, J.; Leblond, F.A.; Pichette, V. Emerging evidence of the impact of kidney disease on drug metabolism and transport. Clin. Pharmacol. Ther. 2008, 83, 898-903.

34. Leblanc, M.; Roy, L.F.; Villeneuve, J.P.; Malo, B.; Pomier-Layrargues, G.; Legault, L. Liver blood flow in chronic hemodialysis patients. Nephron 1996, 73, 396-402. 
35. Spapen, H. Liver perfusion in sepsis, septic shock, and multiorgan failure. Anat. Rec. 2008, 291, 714-720.

36. Soni, N. Wonderful albumin? BMJ 1995, 310, 887-888.

37. Nicholson, J.P.; Wolmarans, M.R.; Park, G.R. The role of albumin in critical illness. Br. J. Anaesth. 2000, 85, 599-610.

38. Kirwan, C.; MacPhee, I.; Philips, B. Using drug probes to monitor hepatic drug metabolism in critically ill patients: Midazolam, a flawed but useful tool for clinical investigation of CYP3A activity? Expert Opin. Drug Metab. Toxicol. 2010, 6, 761-771.

39. Philips, B.J.; Lane, K.; Dixon, J.; Macphee, I. The effects of acute renal failure on drug metabolism. Expert Opin. Drug Metab. Toxicol. 2014, 10, 11-23.

40. Naud, J.; Nolin, T.D.; Leblond, F.A.; Pichette, V. Current understanding of drug disposition in kidney disease. J. Clin. Pharmacol. 2012, 52, 10S-22S.

41. Heinemeyer, G.; Gramm, H.J.; Roots, I.; Dennhardt, R.; Simgen, W. The kinetics of metamizol and its metabolites in critical-care patients with acute renal dysfunction. Eur. J. Clin. Pharmacol. 1993, 45, 445-450.

42. Heinemeyer, G.; Link, J.; Weber, W.; Meschede, V.; Roots, I. Clearance of ceftriaxone in critical care patients with acute renal failure. Intensiv. Care Med. 1990, 16, 448-453.

43. Michaud, J.; Naud, J.; Chouinard, J.; Desy, F.; Leblond, F.A.; Desbiens, K.; Bonnardeaux, A.; Pichette, V. Role of parathyroid hormone in the downregulation of liver cytochrome P450 in chronic renal failure. J. Am. Soc. Nephrol. 2006, 17, 3041-3048.

44. Michaud, J.; Dube, P.; Naud, J.; Leblond, F.A.; Desbiens, K.; Bonnardeaux, A.; Pichette, V. Effects of serum from patients with chronic renal failure on rat hepatic cytochrome P450. Br. J. Pharmacol. 2005, 144, 1067-1077.

45. Krueger, S.K.; Williams, D.E. Mammalian flavin-containingmon-oxygenases: Structure/function, genetic ploymorphism and role in drug metabolism. Pharmacol. Ther. 2005, 106, 357-387.

46. Lohr, J.W.; Willsky, G.R.; Acara, M.A. Renal drug metabolism. Pharmacol. Rev. 1998, 50, 107-141.

47. Kunuzova, O.R.; Bianchi, P.; Pizzinat, N.; Escourrou, G.; Sequelas, M.H.; Parini, A.; Cambon, C. Regulation of JNK/ERK activation, cell apoptosis, and tissue regeneration by monoamine oxidases after renalischemia-reperfusion. FASEB J. 2002, 16, 1129-1131.

48. Andres-Hernando, A.; Altmann, C.; Ahuja, N.; Lanaspa, M.A.; Nemenoff, R.; He, Z.; Ishimoto, T.; Simpson, P.A.; Weiser-Evans, M.C.; Bacalja, J.; et al. Splenectomy exacerbates lung injury after ischemic acute kidney injury in mice. Am. J. Physiol. Ren. Physiol. 2011, 301, F907-F916.

49. Andres-Hernando, A.; Dursun, B.; Altmann, C.; Ahuja, N.; He, Z.; Bhargava, R.; Edelstein, C.E.; Jani, A.; Hoke, T.S.; Klein, C.; et al. Cytokine production increases and cytokine clearance decreases in mice with bilateral nephrectomy. Nephrol. Dial. Transplant. 2012, 27, 4339-4347.

50. Park, S.W.; Chen, S.W.; Kim, M.; Brown, K.M.; Kolls, J.K.; D’Agati, V.D.; Lee, H.T. Cytokines induce small intestine and liver injury after renal ischemia or nephrectomy. Lab. Investig. 2011, 91, 63-84.

51. Park, S.W.; Kim, M.; Kim, J.Y.; Ham, A.; Brown, K.M.; Mori-Akiyama, Y.; Ouellette, A.J.; D’Agati, V.D.; Lee, H.T. Paneth cell-mediated multiorgan dysfunction after acute kidney injury. J. Immunol. 2012, 189, 5421-5433. 
52. Miyazawa, S.; Watanabe, H.; Miyaji, C.; Hotta, O.; Abo, T. Leukocyte accumulation and changes in extra-renal organs during renal ischemia reperfusion in mice. J. Lab. Clin. Med. 2002, 139, 269-278.

53. Fadillioglu, E.; Kurcer, Z.; Parlakpinar, H.; Iraz, M.; Gursul, C. Melatonin treatment against remote organ injury induced by renal ischemia reperfusion injury in diabetes mellitus. Arch. Pharm. Res. 2008, 31, 705-712.

54. Golab, F.; Kadkhodaee, M.; Zahmatkesh, M.; Hedayati, M.; Arab, H.; Schuster, R.; Zahedi, K.; Lentsch, A.B.; Soleimani, M. Ischemic and non-ischemic acute kidney injury cause hepatic damage. Kidney Int. 2009, 75, 783-792.

55. Gurley, B.J.; Barone, G.W.; Yamashita, K.; Polston, S.; Estes, M.; Harden, A. Extrahepatic ischemia-reperfusion injury reduces hepatic oxidative drug metabolism as determined by serial antipyrine clearance. Pharm. Res. 1997, 14, 67-72.

56. Klein, C.L.; Hoke, T.S.; Fang, W.F.; Altmann, C.J.; Douglas, I.S.; Faubel, S. Interleukin-6 mediates lung injury following ischemic acute kidney injury or bilateral nephrectomy. Kidney Int. 2008, 74, 901-909.

57. Muntane-Relat, J.; Ourlin, J.C.; Domergue, J.; Maurel, P. Differential effects of cytokines on the inducible expression of CYP1A1, CYP1A2, and CYP3A4 in human hepatocytes in primary culture. Hepatology 1995, 22, 1143-1153.

58. Chawla, L.S.; Seneff, M.G.; Nelson, D.R.; Williams, M.; Levy, H.; Kimmel, P.L.; Macias, W.L. Elevated plasma concentrations of IL-6 and elevated APACHE II score predict acute kidney injury in patients with severe sepsis. Clin. J. Am. Soc. Nephrol. 2007, 2, 22-30.

59. Liu, K.D.; Altmann, C.; Smits, G.; Krawczeski, C.D.; Edelstein, C.L.; Devarajan, P.; Faubel, S. Serum interleukin-6 and interleukin-8 are early biomarkers of acute kidney injury and predict prolonged mechanical ventilation in children undergoing cardiac surgery: A case-control study. Crit. Care 2009, doi:10.1186/cc7940.

60. Liu, K.D. Impact of acute kidney injury on lung injury. Am. J. Physiol. Lung Cell. Mol. Physiol. 2009, doi:10.1152/ajplung.90541.2008.

61. Mastorakos, G.; Chrousos, G.P.; Weber, J.S. Recombinant interleukin-6 activates the hypothalamic-pituitary-adrenal axis in humans. J. Clin. Endocrinol. Metab. 1993, 77, 1690-1694.

62. Lee, A.K.; Lee, J.H.; Kwon, J.W.; Kim, W.B.; Kim, S.G.; Kim, S.H.; Lee, M.G. Pharmacokinetics of clarithromycin in rats with acute renal failure induced by uranyl nitrate. Biopharm. Drug Dispos. 2004, 25, 273-282.

63. Lee, J.H.; Lee, M.G. Effects of acute renal failure on the pharmacokinetics of telithromycin in rats: Negligible effects of increase in CYP3A1 on the metabolism of telithromycin. Biopharm. Drug Dispos. 2007, 28, 157-166.

64. Venkatesh, P.; Harisudhan, T.; Choudhury, H.; Mullangi, R.; Srinivas, N.R. Pharmacokinetics of etoposide in rats with uranyl nitrate (UN)-induced acute renal failure (ARF): Optimization of the duration of UN dosing. Eur. J. Drug Metab. Pharmacokinet. 2007, 32, 189-196.

65. Yoshitani, T.; Yagi, H.; Inotsume, N.; Yasuhara, M. Effect of experimental renal failure on the pharmacokinetics of losartan in rats. Biol. Pharm. Bull. 2002, 25, 1077-1083.

66. Okabe, H.; Yano, I.; Hashimoto, Y.; Saito, H.; Inui, K. Evaluation of increased bioavailability of tacrolimus in rats with experimental renal dysfunction. J. Pharm. Pharmacol. 2002, 54, 65-70. 
67. Izuwa, Y.; Kusaba, J.; Horiuchi, M.; Aiba, T.; Kawasaki, H.; Kurosaki, Y. Comparative study of increased plasma quinidine concentration in rats with glycerol- and cisplatin-induced acute renal failure. Drug Metab. Pharmacokinet. 2009, 24, 451-457.

68. Shibata, N.; Inoue, Y.; Fukumoto, K.; Nishimura, A.; Fukushima, K.; Yoshikawa, Y.; Spiteller, G.; Takada, K. Evaluation of factors to decrease bioavailability of cyclosporin A in rats with gentamicin-induced acute renal failure. Biol. Pharm. Bull. 2004, 27, 384-391.

69. Karim, M.S.; Wood, R.F.; Dawnay, A.B.; Fulton, P.A. The effect of renal ischemia on cyclosporine clearance in rabbits. Transplantation 1990, 49, 500-502.

70. Okabe, H.; Higashi, T.; Ohta, T.; Hashimoto, Y. Intestinal absorption and hepatic extraction of propranolol and metoprolol in rats with bilateral ureteral ligation. Biol. Pharm. Bull. 2004, 27, $1422-1427$.

71. Tanabe, H.; Taira, S.; Taguchi, M.; Hashimoto, Y. Pharmacokinetics and hepatic extraction of metoprolol in rats with glycerol-induced acute renal failure. Biol. Pharm. Bull. 2007, 30, 552-555.

72. Okabe, H.; Hasunuma, M.; Hashimoto, Y. The hepatic and intestinal metabolic activities of P450 in rats with surgery- and drug-induced renal dysfunction. Pharm. Res. 2003, 20, 1591-1594.

73. Yu, S.Y.; Chung, H.C.; Kim, E.J.; Kim, S.H.; Lee, I.; Kim, S.G.; Lee, M.G. Effects of acute renal failure induced by uranyl nitrate on the pharmacokinetics of intravenous theophylline in rats: The role of CYP2E1 induction in 1,3-dimethyluric acid formation. J. Pharm. Pharmacol. 2002, 54, $1687-1692$.

74. Srinivas, N.R. Pharmacokinetics of tolbutamide in acute renal failure induced by glycerol: Speculative thoughts and perspectives. Drug Metab. Pharmacokinet. 2011, 26, 216-217.

75. Wang, B.Y.; Li, Q.X.; Li, J.; Xie, X.F.; Ao, Y.; Ai, Y.X. Hepatotoxicity and gene expression down-regulation of CYP isozymes caused by renal ischemia/reperfusion in the rat. Exp. Toxicol. Pathol. 2009, 61, 169-176.

76. Chung, H.C.; Kim, S.H.; Lee, M.G.; Kim, S.G. Increase in urea in conjunction with L-arginine metabolism in the liver leads to induction of cytochrome P450 2E1 (CYP2E1): The role of urea in CYP2E1 induction by acute renal failure. Drug Metab. Dispos. 2002, 30, 739-746.

77. Kunihara, M.; Nagai, J.; Murakami, T.; Takano, M. Renal excretion of rhodamine 123, a P-glycoprotein substrate, in rats with glycerol-induced acute renal failure. J. Pharm. Pharmacol. 1998, 50, 1161-1165.

78. Murakami, T.; Yumoto, R.; Nagai, J.; Takano, M. Factors affecting the expression and function of P-glycoprotein in rats: Drug treatments and diseased states. Die Pharm. 2002, 57, 102-107.

79. Yamaguchi, H.; Yano, I.; Saito, H.; Inui, K. Effect of cisplatin-induced acute renal failure on bioavailability and intestinal secretion of quinolone antibacterial drugs in rats. Pharm. Res. 2004, 21, 330-338.

80. Chan, L.M.; Lowes, S.; Hirst, B.H. The ABCs of drug transport in intestine and liver: Efflux proteins limiting drug absorption and bioavailability. Eur. J. Pharm. Sci. 2004, 21, 25-51.

81. Schneider, R.; Sauvant, C.; Betz, B.; Otremba, M.; Fischer, D.; Holzinger, H.; Wanner, C.; Galle, J.; Gekle, M. Downregulation of organic anion transporters OAT1 and OAT3 correlates with impaired secretion of para-aminohippurate after ischemic acute renal failure in rats. Am. J. Physiol. Ren. Physiol. 2007, 292, F1599-F1605. 
82. Sun, H.; Frassetto, L.; Benet, L.Z. Effects of renal failure on drug transport and metabolism. Pharmacol. Ther. 2006, 109, 1-11.

83. Shi, J.; Montay, G.; Chapel, S.; Hardy, P.; Barrett, J.S.; Sack, M.; Marbury, T.; Swan, S.K.; Vargas, R.; Leclerc, V.; et al. Pharmacokinetics and safety of the ketolide telithromycin in patients with renal impairment. J. Clin. Pharmacol. 2004, 44, 234-244.

84. Nolin, T.D.; Appiah, K.; Kendrick, S.A.; Le, P.; McMonagle, E.; Himmelfarb, J. Hemodialysis acutely improves hepatic CYP3A4 metabolic activity. J. Am. Soc. Nephrol. 2006, 17, 2363-2367.

85. Gertz, M.; Harrison, A.; Houston, J.B.; Galetin, A. Prediction of human intestinal first-pass metabolism of $25 \mathrm{CYP} 3 \mathrm{~A}$ substrates from in vitro clearance and permeability data. Drug Metab. Dispos. 2010, 38, 1147-1158.

(C) 2014 by the authors; licensee MDPI, Basel, Switzerland. This article is an open access article distributed under the terms and conditions of the Creative Commons Attribution license (http://creativecommons.org/licenses/by/3.0/). 